\title{
Spectral Content of a Single Non-Brownian Trajectory
}

\author{
Diego Krapf, ${ }^{1,2}$ Nils Lukat, ${ }^{3}$ Enzo Marinari, ${ }^{4,5}$ Ralf Metzler, ${ }^{6}$ Gleb Oshanin, ${ }^{7, *}$ Christine Selhuber-Unkel, ${ }^{3}$ \\ Alessio Squarcini, ${ }^{8,9}$ Lorenz Stadler, ${ }^{10}$ Matthias Weiss, ${ }^{10}$ and Xinran $\mathrm{Xu}^{1}$ \\ ${ }^{1}$ Department of Electrical and Computer Engineering, Colorado State University, \\ Fort Collins, Colorado 80523, USA \\ ${ }^{2}$ School of Biomedical Engineering, Colorado State University, Fort Collins, Colorado 80523, USA \\ ${ }^{3}$ Institute for Materials Science, Biocompatible Nanomaterials, University of Kiel, \\ Kaiserstrasse 2, 24143 Kiel, Germany \\ ${ }^{4}$ Dipartimento di Fisica, Sapienza Università di Roma, P.le A. Moro 2, I-00185 Roma, Italy \\ ${ }^{5}$ INFN, Sezione di Roma 1 and Nanotech-CNR, UOS di Roma, P.le A. Moro 2, I-00185 Roma, Italy \\ ${ }^{6}$ Institute for Physics and Astronomy, University of Potsdam, \\ Karl-Liebknecht-Strasse 24/25, 14476 Potsdam-Golm, Germany \\ ${ }^{7}$ Sorbonne Université, CNRS, Laboratoire de Physique Théorique de la Matière Condensée (UMR 7600), \\ 4 Place Jussieu, 75252 Paris Cedex 05, France \\ ${ }^{8}$ Max-Planck-Institut für Intelligente Systeme, Heisenbergstrasse 3, 70569, Stuttgart, Germany \\ ${ }^{9} I V$. Institut für Theoretische Physik, Universität Stuttgart, Pfaffenwaldring 57, 70569 Stuttgart, Germany \\ ${ }^{10}$ Experimental Physics I, University of Bayreuth, D-95440 Bayreuth, Germany
}

(Received 29 September 2018; revised manuscript received 22 December 2018; published 31 January 2019)

Time-dependent processes are often analyzed using the power spectral density (PSD) calculated by taking an appropriate Fourier transform of individual trajectories and finding the associated ensemble average. Frequently, the available experimental datasets are too small for such ensemble averages, and hence, it is of a great conceptual and practical importance to understand to which extent relevant information can be gained from $S(f, T)$, the PSD of a single trajectory. Here we focus on the behavior of this random, realization-dependent variable parametrized by frequency $f$ and observation time $T$, for a broad family of anomalous diffusions-fractional Brownian motion with Hurst index $H$-and derive exactly its probability density function. We show that $S(f, T)$ is proportional-up to a random numerical factor whose universal distribution we determine-to the ensemble-averaged PSD. For subdiffusion $(H<1 / 2)$, we find that $S(f, T) \sim A / f^{2 H+1}$ with random amplitude $A$. In sharp contrast, for superdiffusion $(H>1 / 2) S(f, T) \sim B T^{2 H-1} / f^{2}$ with random amplitude $B$. Remarkably, for $H>1 / 2$ the PSD exhibits the same frequency dependence as Brownian motion, a deceptive property that may lead to false conclusions when interpreting experimental data. Notably, for $H>1 / 2$ the PSD is ageing and is dependent on $T$. Our predictions for both sub- and superdiffusion are confirmed by experiments in live cells and in agarose hydrogels and by extensive simulations.

DOI: 10.1103/PhysRevX.9.011019

\section{INTRODUCTION}

The power spectral density of any time-dependent process $X_{t}$ is a fundamental feature of its spectral content, dynamical behavior, and temporal correlations [1]. It is an important measure for various processes across many

*oshanin@lptmc.jussieu.fr

Published by the American Physical Society under the terms of the Creative Commons Attribution 4.0 International license. Further distribution of this work must maintain attribution to the author(s) and the published article's title, journal citation, and DOI.
Subject Areas: Biological Physics, Interdisciplinary Physics, Statistical Physics disciplines, including loudness of musical recording [2,3], evolution of climate data [4], time gaps between large earthquakes [5], retention times of chemical tracers in groundwater [6], noise in graphene devices [7], fluorescence intermittency in nanosystems [8], current fluctuations in nanoscale electrodes [9], stochastic processes with random reset [10], some extremal properties of Brownian motion [11], diffusion in strongly disordered systems $[12,13]$, and ionic currents across nanopores [14], to name a few diverse examples.

In its standard definition, the power spectral density (PSD) is the Fourier transform of the autocorrelation function of $X_{t}$ over an infinitely large observation time $T$; i.e., it is an ensemble-averaged property taken in the 
limit $T \rightarrow \infty$. In many situations, however, one cannot create a sufficiently large statistical sample to achieve a reliable ensemble average, and even though the limit $T \rightarrow \infty$ can be formally taken in mathematical expressions, it cannot be reached in experiments. Instead, one often deals with either a single or a few individual finite-length realizations of the process, particularly, in experimental data dealing with in vivo systems [15], climate change [16], or financial markets [17]. In this regard, a question of immense conceptual and practical importance is whether one can learn relevant information about the system from the PSD of just a single or a few finite-length realizations.

Several recent studies examined the PSD from such single-trajectory data. Power spectra of individual time series were examined for a stochastic model describing blinking quantum dots $[18,19]$ and also for single-particle tracking experiments with tracers in artificially crowded fluids [20]. Notably, the scaling exponent of the power spectrum computed from a few single-trajectory PSDs remains very stable. In addition, the power spectra of the velocity of motile amoeba revealed a robust large- $f$ asymptotic behavior of the form $1 / f^{2}$ for all measured individual trajectories $[21,22]$.

Without a solid mathematical theory, these observations [18-22] may be considered merely as curious coincidences. However, in a recent work [23] (see also the perspective [24]), it was proven that for standard Brownian motion, the single-trajectory PSD $S(f, T)$ in the large- $f$ limit and at finite $T$ exhibits the same $f$ dependence as its traditional ensemble-average counterpart. This mathematical prediction was fully corroborated by numerical simulations and experiments with polystyrene beads in aqueous solution [23].

Despite its ubiquitous appearance in nature [25], Brownian motion is just a particular example of a stochastic process, and there is no evidence that the same behavior should hold for other naturally occurring transport processes. In this regard, it seems highly desirable to have analogous proof for anomalous diffusion, with meansquared displacement (MSD)

$$
\left\langle X_{t}^{2}\right\rangle \sim t^{\alpha},
$$

and anomalous-diffusion exponent $\alpha \neq 1$, where the brackets here and henceforth denote the average over the statistical ensemble.

Such processes are widely observed in soft matter, condensed matter, and biological systems, e.g., diffusion in viscoelastic and crowded systems, the motion of proteins $[15,26]$ or submicron tracers in living cells $[27,28]$, in artificially crowded liquids $[29,30]$, telomere diffusion in the cell nucleus [31], diffusion in disordered media [32], dynamics of ultracold atoms [33], and in lipid membranes [34-37]. Anomalous diffusion is also found in other systems, including heartbeat intervals [38], DNA sequence landscapes [39], and even in the daily fluctuations of climate variables [40] and economic markets [17].
Here we calculate exactly for any $T, f$, and $\alpha$ the full probability density function (PDF) of a single-trajectory PSD $S(f, T)$ - a random, realization-dependent variablefor the widely observed process of fractional Brownian motion (FBM) [41,42] (see also Sec. II for more details). Analogous to the parental FBM process, the PDF of the PSD of its individual realizations appears to be entirely characterized by its two first moments: We thus derive an explicit expression for the ensemble-averaged PSD

$$
\mu=\mu(f, T)=\langle S(f, T)\rangle,
$$

the first moment of the PDF, which is a standard property, and we also go beyond the textbook definition and determine its variance

$$
\sigma^{2}=\sigma^{2}(f, T)=\left\langle S^{2}(f, T)\right\rangle-\langle S(f, T)\rangle^{2} .
$$

These results permit us to quantify the effective broadness of the PDF via its coefficient of variation $\gamma=\sigma / \mu$. We realize that (for any $f$ and $T$ and regardless of the value of the anomalous-diffusion exponent) $\gamma$ always exceeds the value 1 such that the standard deviation $\sigma$ of the singletrajectory PSD is always greater than its ensemble-averaged value $\mu$. This observation implies that the PDF is broad and cannot be characterized exhaustively solely by its first moment, which justifies a posteriori our quest for the form of the full PDF. Moreover, we find that the value achieved by $\gamma$ in the limit $f T \rightarrow \infty$ is very meaningful, and on this basis, we offer a novel and very robust criterion, which permits us to prove the anomalous character of random motion in situations where the analysis of the MSD deduced from experimental data leads to ambiguous conclusions.

Our theoretical analysis then culminates at the observation that for sufficiently large values of $f$ a single-trajectory PSD $S(f, T)$ is linearly proportional (with a universal, dimensionless, random proportionality factor) to its mean value $\mu$, which embodies the full dependence on $T$ and $f$. This finding generalizes the previous observation made in Ref. [23] for standard Brownian motion to a wide class of anomalous diffusion. Here, however, the value of the anomalous-diffusion exponent appears to be crucially important: For $\alpha<1$ (subdiffusion), the PSD attains a stationary form $1 / f^{\alpha+1}$ for sufficiently large $f$ and $T$, while in the superdiffusive case $(\alpha>1)$, the leading behavior of the PSD is given by $T^{\alpha-1} / f^{2}$. I.e., the PSD is ageing and is deceivingly proportional to $1 / f^{2}$, where the exponent 2 characterizing the $f$ dependence is the same as for standard Brownian motion $(\alpha=1)$ [23] regardless of the actual value of $\alpha>1$. As a consequence, one should exercise a great deal of care in the interpretation of the data for superdiffusive motion and rather concentrate on the ageing behavior ( $T$ dependence) than on the $f$ dependence, of $S(f, T)$. 
Further on, we compare a variety of our analytical predictions against the corresponding analysis of singletrajectory data garnered from experiments in quite diverse systems: the dynamics of telomeres in the nucleus of live cells, polystyrene microspheres in agarose hydrogels, and motile Acanthamoeba castellanii and their intracellular vacuoles. Some features of the predicted behavior of the PDF, which we cannot access in experiments, are also verified by an extensive numerical analysis. As we show, our analytical predictions on the $f$ dependence of the PSD in the subdiffusive case, ageing behavior, and the deceptive $1 / f^{2}$ dependence in the superdiffusive case, as well as the corresponding distributions of the universal random amplitude are fully in line with experimental observations in biologically relevant systems.

We remark that the four systems used in our experimental analysis are just a few particular examples of systems with a FBM-type dynamics. In general, FBM encompasses a broad range of naturally occurring processes with continuous paths and long-ranged temporal correlations, which entail both sub- and superdiffusive behaviors, depending on whether the increments of particle displacement are negatively or positively correlated. In particular, physically FBM processes describe the overdamped, antipersistent motion of particles in viscoelastic environments $[20,42,43]$, as well as the persistent superdiffusion in actively driven systems [44]. The characteristic antipersistent signature of subdiffusive FBM was identified in the dynamics of chromosomal loci and ribonucleic-acidprotein particles in live bacterial cells [45], lipid granules in yeast cells in the millisecond range [27], tracer beads in wormlike micellar solutions [29], lipid molecules in dilute bilayer membranes in supercomputing studies [35,46,47], and chromatin in Langevin dynamics simulations [48]. With different observables, FBM-type motion was further identified in the dynamics of chromosomal telomeres in living U2OS cells [49] and nanosized particles in crowded dextran solutions $[20,30,50]$.

FBM is thus a very generic stochastic process, and it combines both subdiffusive and superdiffusive motion in a common framework; therefore, we regard it here as the first prototype example for the study of single-trajectory PSD. Of course, FBM does not cover all possible kinds of anomalous diffusion [42]. Moreover, in some instances, FBM dominates the dynamics of a system at intermediate timescales, and it is tempered to become standard diffusion at longer times, or dynamical transitions between different types of FBM may take place [51,52]. A systematic analysis of other representative examples of anomalous diffusion, combinations of different anomalous diffusions, and processes with dynamical transitions between different types of behavior is thus ultimately necessary in order to attain a full understanding of the spectral content of a single-trajectory PSD. In turn, such an analysis will provide robust criteria eventually permitting us to distinguish between different types of random motion. Our work thus represents an essential first step in this direction.

The outline of this paper is as follows: In Sec. II, we describe the statistical properties of fractional Brownian motion, present the definitions of the random variables of interest here, namely, the power spectral density of individual trajectories of FBM in the case of one-dimensional dynamics, as well as for a more general case of a $d$-dimensional dynamics with projections on the coordinate axes. We also present the definition of the moment-generating function of the PSD of individual FBM trajectories. The desired PDF then follows from the moment-generating function upon a mere inversion of the Laplace transform. In Sec. III, we describe our experimental systems which exhibit anomalous, nonBrownian dynamics and also briefly recall how both the MSD and the PSD can be deduced from the experimental data. At the end of this section, we also describe the algorithm of our numerical analysis. Section IV presents our main exact analytical results and a discussion of their asymptotic behavior. On this basis, we formulate here a robust, novel criterion which will permit us to prove the anomalous or normal (standard Brownian) character of dynamics. This criterion is based on a statistical sample and is validated by numerical simulations. Further on, in Sec. V, we compare our analytical predictions against the results of simulations and experimental data garnered from experiments performed for four different systems exhibiting an anomalous behavior. In Sec. VI, we present a brief summary of our results and a perspective.

\section{FRACTIONAL BROWNIAN MOTION AND ITS POWER SPECTRAL DENSITIES}

FBM is a Gaussian stochastic process, and hence, it is entirely characterized by its first moment and the covariance, which defines its autocorrelation at two different time instants $t_{1}$ and $t_{2}$. FBM has zero mean value, and its covariance function is given by

$$
\left\langle X_{t_{1}} X_{t_{2}}\right\rangle=D\left[t_{1}^{2 H}+t_{2}^{2 H}-\left|t_{1}-t_{2}\right|^{2 H}\right],
$$

where $H \in(0,1)$ is the so-called Hurst index [41,42]. Comparing the expression in Eq. (4) for $t_{1}=t_{2}$ with Eq. (1), one infers that for FBM the anomalous-diffusion exponent $\alpha$ is simply related to $H, \alpha=2 H$.

Standard Brownian motion, on which the analysis in Ref. [23] is concentrated, is recovered for a particular case $H=1 / 2$ only. In this case, the increments of the process are independent, and $D$ is the standard diffusion coefficient. When $H>1 / 2$ (corresponding to $\alpha>1$ ), the increments are positively correlated, such that if there is an increasing pattern in the previous steps, it is likely that the current step will be increasing as well, resulting ultimately in a superdiffusive motion. For $H<1 / 2$, the increments are negatively correlated, such that it is most likely that after an increasing step a decreasing one will follow, entailing a subdiffusive motion. In the two latter cases, $D$ can 
be thought of as a proportionality factor with units length ${ }^{2} /$ time $^{2 H}$. PSD

We focus here on the single-component single-trajectory

$$
S(f, T)=\frac{1}{T} \mid\left.\int_{0}^{T} \exp (\text { ift }) X_{t} d t\right|^{2},
$$

which is a random variable dependent on a given realization $X_{t}$ of a one-dimensional FBM and is parametrized by the observation time $T$ and the frequency $f$. For its generalization over the $d$-dimensional case, we represent a trajectory $\mathbf{R}_{t}$ of a $d$-dimensional FBM as $\mathbf{R}_{t}=\left\{X_{t}^{(1)}, X_{t}^{(2)}, \ldots, X_{t}^{(d)}\right\}$ [53]. Here, $X_{t}^{(j)}$ is the projection of $\mathbf{R}_{t}$ onto the axis $x_{j}$ and is statistically independent of other components. With this definition, we consider the $k$-component version of a $d$-dimensional single-trajectory PSD,

$$
S_{k}(f, T)=\frac{1}{T} \sum_{j=1}^{k} \mid\left.\int_{0}^{T} \exp (\text { ift }) X_{t}^{(j)} d t\right|^{2},
$$

where $k=1, \ldots, d$ is the number of the tracked components. For $k=1$, Eq. (6) reduces to $S(f, T)$.

We note that the standard textbook definition of the PSD is based on the ensemble-averaged expressions in Eqs. (5) and (6). Our aim here is much more ambitious: We proceed to calculate exactly the full PDF of the random variable $S_{k}(f, T)$ for arbitrary $H, k, f$, and $T$. This can be done rather straightforwardly if we manage to determine the moment-generating function of the random variable $S_{k}(f, T)$ defined formally by

$$
\Phi_{\lambda}=\left\langle\exp \left(-\frac{\lambda}{T} \sum_{j=1}^{k}\left|\int_{0}^{T} \exp (i f t) X_{t}^{(j)} d t\right|^{2}\right)\right\rangle,
$$

with $\lambda \geq 0$. The desired PDF $P\left[S_{k}\right]$ follows from Eq. (7) by a mere inversion of the Laplace transform with respect to the parameter $\lambda$. Exact results for both the momentgenerating function and the PDF, as well as asymptotic expressions of the first two moments of the PDF are presented in Sec. IV. Details of the derivations, which are rather lengthy, and also quite cumbersome exact expressions for the first two moments of the PDF [valid for arbitrary values of the parameters $f$ and $T$ and for arbitrary $H \in(0,1)]$ are presented in the Supplemental Material [54].

\section{EXPERIMENT AND NUMERICAL ANALYSES}

\section{A. Experimental systems}

We study experimentally the dynamics of polystyrene microspheres in agarose hydrogels, telomeres in the nucleus of live cells, motile Acanthamoeba castellanii amoeba, and their intracellular vacuoles.

\section{Agarose hydrogel}

We record the motion of 50-nm microspheres in agarose hydrogel. A $1.5 \%$ agarose gel is prepared from agarose powder (Cat. 20-102GP, Genesee Scientific, San Diego, CA) without further purification by dissolving it in phosphate-buffered saline. Carboxylate-modified polystyrene microspheres with 50-nm nominal diameter (Cat. PC02002, Bangs Laboratories, Fishers, IN) are first heated to $60{ }^{\circ} \mathrm{C}$ in $0.5 \%$ Tween 20 and introduced into the agarose solution also at $60^{\circ} \mathrm{C}$. The agarose and microsphere solution is allowed to mix at $60^{\circ} \mathrm{C}$ for $15 \mathrm{~min}$ and then transferred to a hot glass-bottom Petri dish and left to slowly cool to room temperature.

The microspheres are imaged in an inverted microscope equipped with a $40 \times$ objective (Olympus PlanApo, N.A. 0.95) and a scientific CMOS camera (Andor Zyla 4.2) operated at 71 frames/s. The first 2048 images are used for further tracking and analysis. Tracking of the microspheres in the plane is performed in LABVIEW using a crosscorrelation-based tracking algorithm [55]. Immobile particles and particles that exhibit very little motion are discarded. A total of 20 trajectories are analyzed in terms of their PSD.

\section{Telomeres}

Trajectories of telomeres in the nucleus of untreated U2OS cells are acquired at 8 frames/s and evaluated as described before [56]. It was shown previously that the time-averaged mean-squared displacement (TA MSD) of these trajectories features a FBM-like subdiffusive scaling for short and intermediate times with a mean exponent $\langle\alpha\rangle \approx 0.5$. From these previously analyzed data, we select 19 individual trajectories, each 2500 frames in length with scaling exponents in the range $\alpha=0.5 \pm 0.05$ for the PSD analysis.

\section{Amoeba and intracellular vacuoles}

Trajectories of amoeba and intracellular vacuoles are recorded using A. castellanii cultured as previously described [44]. Imaging is done using a Hamamatsu ORCA ER2 camera on an Olympus IX71 microscope, and the images are recorded with the MATLAB Image Acquisition Toolbox (Mathworks, Inc.) at 9 frames/s. In addition, every $2 \mathrm{~s}$, the image is segmented using an edge detection algorithm in MATLAB, and the center of mass of the amoeba is calculated. To record over long periods, the amoeba is kept in the center of the image by automatically moving along a scanning stage (Märzhäuser, SCAN IM $112 \times 74)$. In addition, the position of intracellular vacuoles is detected using a home-written segmentation algorithm in MATLAB. In brief, first edge detection is carried out, followed by a Hough transformation to find circles and an algorithm to verify the vacuoles by their light edge. The center of the circles in the images is determined and gives 
the position of the vacuoles. All trajectories are optically verified. Previously, it was shown that the vacuole intracellular motion within A. castellanii is superdiffusive [44].

Four individual amoeba trajectories, each consisting of 16384 frames, are analyzed in terms of their PSD. Short vacuole trajectories are discarded, and 50 vacuoles trajectories, all from the same cell, are analyzed. In order to avoid differences in trajectory lengths, only the first 2048 frames in each vacuole trajectory are used in the analysis.

\section{B. Mean-squared displacement}

The time-averaged MSD of individual trajectories $X_{p}$, where $p$ is an appropriately discretized time variable, is defined as (see, e.g., Ref. [42])

$$
\overline{\delta^{2}(\Delta)}=\frac{1}{N-\Delta} \sum_{p=1}^{N-\Delta}\left(X_{p+\Delta}-X_{p}\right)^{2} .
$$

This property is computed in MATLAB as a function of the lag time $\Delta$ for all analyzed trajectories. The MSD of the representative microspheres, amoeba, and vacuole trajectories along with their ensemble mean are shown in Fig. 1. The MSD from the analyzed telomere trajectories were previously reported [56]. The anomalous exponent as obtained from the MSD for microspheres is $\alpha_{S}=0.87$ for short times and $\alpha_{L}=0.61$ for longer times [Fig. 1(a)], for telomeres $\alpha=0.5$ (see Ref. [56]), for vacuoles $\alpha=$ 1.33 [Fig. 1(b)], and for the amoeba $\alpha=1.97$ [Fig. 1(c)].

\section{PSD analysis}

Single- and two-component PSDs of individual trajectories [as defined in Eqs. (5) and (6), respectively] are obtained in MATLAB from the Fourier-transformed components $X_{t}^{(1)}$ and $X_{t}^{(2)}$ of three-dimensional trajectories. Care is taken that all trajectories of the same type include the same number of data points and the same frame rate.

For analyzing the fluctuations of the PSD, i.e., to obtain the empirical distributions of the amplitudes of the PSD for sub- and superdiffusive cases, the gross scaling $1 / f^{\beta}$ is obtained from the ensemble-averaged PSD, where $\beta=2 H+1$ for the subdiffusive cases (microspheres and telomeres) and $\beta=2$ for the superdiffusive ones (vacuoles). From these datasets, we extract the values $A^{\prime}=S(f, T) f^{\beta}$ [see Eq. (12) below] in the following frequency ranges: (i) $11 \mathrm{~Hz}<f<87 \mathrm{~Hz}$ for microspheres, (ii) $1 \mathrm{~Hz}<f<10 \mathrm{~Hz}$ for telomeres, and (iii) $1 \mathrm{~Hz}<f<5 \mathrm{~Hz}$ for vacuoles. We do not extract the fluctuations of the amoeba because only four trajectories are used. Then, we normalize the fluctuations according to $A=A^{\prime} /\left\langle A^{\prime}\right\rangle$. The same procedure is followed to obtain $B$ [see Eq. (14) below] for the vacuoles. These data are then compared to the theoretical predictions as described in Sec. IV below.

\section{Numerical algorithms}

The numerical simulations of FBM are far more complicated than the ones used, e.g., for a standard Brownian motion. FBM is not a Markov process and has long-range correlations. In order to reproduce FBM numerically, we use the exact Davies-Harte circulant method (see, e.g., Refs. [57-61]). Because of the use of the fast Fourier transform, the required CPU time for reproducing a $T$-steps trajectory is of order $T \log (T)$ (and not of order $T^{2}$ as a naive approach would give). The Davies-Harte approach is a very powerful exact method, and for the samples of the size we use, its running time is comparable to the one of effective approximate methods [60]. We use trajectories of $T=2^{21}$ to $T=2^{23}$ discrete time steps. The total CPU time we use for all numerical runs is of the order of a few months of one core of Intel(R) Xeon(R) CPU E5-2620 0 @ $2.00 \mathrm{GHz}$.

\section{ANALYTICAL PREDICTIONS}

Our first step consists of calculating the momentgenerating function $\Phi_{\lambda}$ of the $k$-component singletrajectory PSD [see Eq. (6)] defined in Eq. (7). We obtain
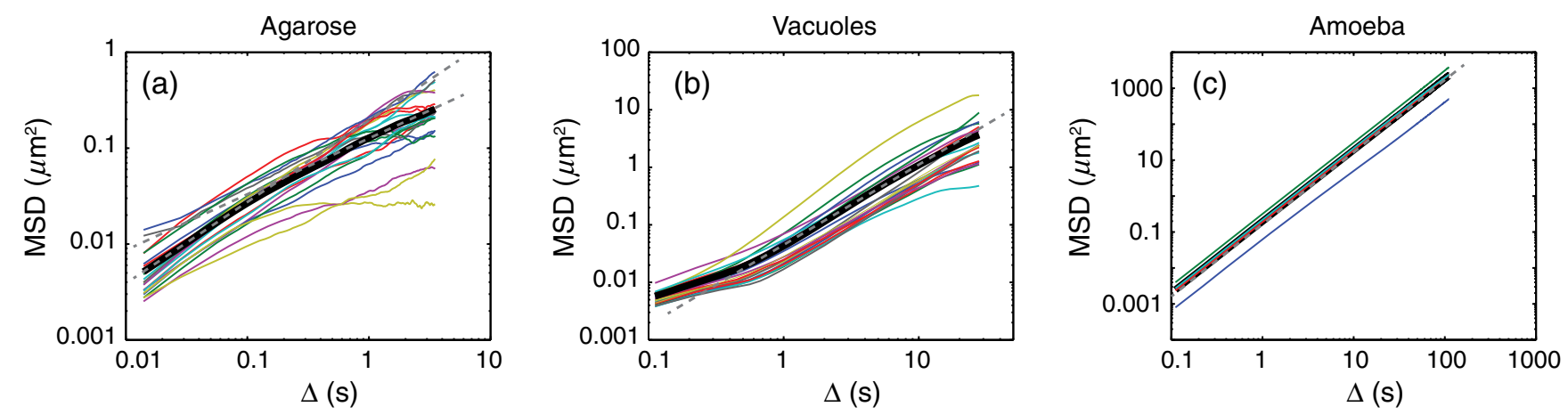

FIG. 1. MSD analysis. (a) TA MSD Eq. (8) as a function of lag time $\Delta$ for 20 individual microsphere trajectories together with their ensemble average. (b) TA MSD for 20 individual representative vacuole trajectories together with the ensemble average from 50 trajectories. (c) TA MSD for four individual amoeba trajectories together with their ensemble average. In all three panels, the thick black lines show the ensemble averages, and the gray dashed lines show power-law behavior MSD, which scales as $\Delta^{\alpha}$. 
(see the Supplemental Material [54] for the details of the derivation)

$$
\Phi_{\lambda}=\left[1+2 \mu \lambda+\left(2-\gamma^{2}\right) \mu^{2} \lambda^{2}\right]^{-k / 2},
$$

where $\mu$ is the first moment of a single-component singletrajectory PSD Eq. (2), $\sigma^{2}$ is the variance of this random variable Eq. (3), and $\gamma=\sigma / \mu$ is the coefficient of variation of the PDF of a single-component single-trajectory PSD $S(f, T)$ Eq. (5). Inverting the Laplace transform with respect to $\lambda$, we readily obtain the $\mathrm{PDF}$ of $S_{k}(f, T)$,

$$
\begin{aligned}
P\left[S_{k}(f, T)=S\right]= & \frac{\sqrt{\pi}}{2^{\frac{k-1}{2}} \Gamma(k / 2) \sqrt{2-\gamma^{2}}\left(\gamma^{2}-1\right)^{\frac{k-1}{4}}} \\
& \times \frac{S^{\frac{k-1}{2}}}{\mu^{\frac{k+1}{2}}} \exp \left(-\frac{1}{2-\gamma^{2}} \frac{S}{\mu}\right) I_{\frac{k-1}{2}}\left(\frac{\sqrt{\gamma^{2}-1}}{2-\gamma^{2}} \frac{S}{\mu}\right),
\end{aligned}
$$

where $I_{\nu}(z)$ is the modified Bessel function of the first kind. We emphasize that the expressions in Eqs. (9) and (10) are exact and hold for any $f, T$, and also for any value of the Hurst index $H$. We note that $\Phi_{\lambda}$ and $P\left[S_{k}(f, T)=S\right]$ are entirely defined by the first two moments of $S(f, T)$, and hence, all higher moments of the $k$-component singletrajectory $\operatorname{PSD} S_{k}(f, T)$ can be expressed solely through the first two moments of $S(f, T)$. This fact is a direct consequence of the Gaussian nature of the parental process $X_{t}$ and suggests, in turn, that the expressions in Eqs. (9) and (10) may hold, in general, for arbitrary Gaussian processes, not necessarily for the FBM only. The dependence of $\mu, \sigma^{2}$, and hence, of $\gamma$ on the characteristic parameters will depend, of course, on the case at hand.

For FBM processes, the exact dependence of $\mu, \sigma$, and hence, of $\gamma$ on $f$ and $T$ for any value of $H \in(0,1)$ is presented in the Supplemental Material [54]. Below, we discuss their rather complex behavior focusing first on the coefficient of variation $\gamma$, which characterizes the effective broadness of the PDF in Eq. (10).

The coefficient of variation $\gamma$, which enters Eqs. (9) and (10), is a dimensionless numerical factor that depends on $f$ and $T$ only through the function $\omega=f T$. Figure 2 shows $\gamma$ as a function of $\omega$ for six different Hurst indices spanning the range $1 / 4 \leq H \leq 7 / 8$. The behavior of $\gamma$ has several characteristic features, which can be clearly observed in Fig. 2:

(i) In the limit $\omega \rightarrow 0$, the coefficient $\gamma$ tends to the universal value $\sqrt{2}$, regardless of the value of $H$. Next, $\gamma$ is an oscillatory function of $\omega$, and the oscillations are prominent at moderate values of $\omega$. In the limit $\omega \gg 1$, the oscillatory terms fade out, and $\gamma$ is given by very simple asymptotic formula (see the Supplemental Material [54] for derivation)

$$
\gamma \sim\left[1+\left(1+c_{H} \omega^{1-2 H}\right)^{-2}\right]^{1 / 2},
$$

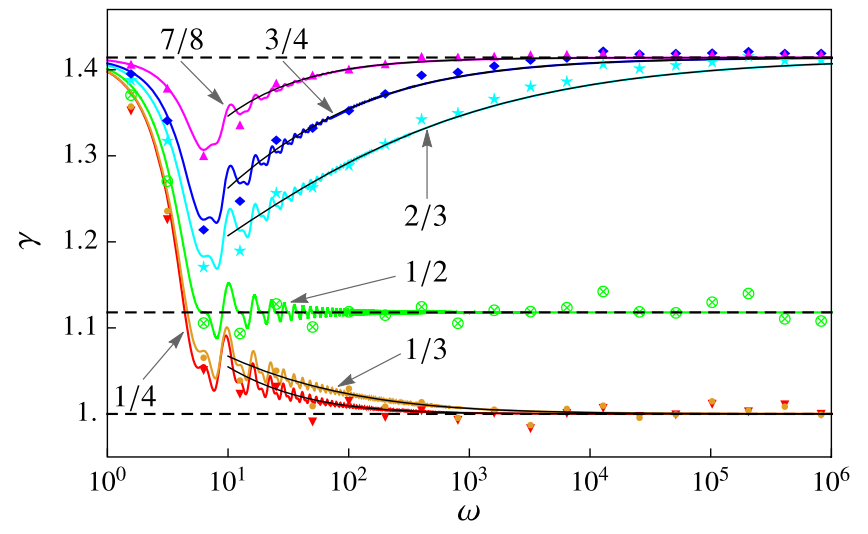

FIG. 2. Coefficient of variation $\gamma$ as a function of $\omega=f T$. Colored solid curves represent exact values of $\gamma$ (arrows indicate the corresponding values of $H$ ) defined by Eq. (47) in the Supplemental Material [54], while thin solid curves depict the asymptotic expression in Eq. (11). Horizontal dashed lines correspond to $\sqrt{2}$ (top), $\sqrt{5} / 2$ (middle), and 1 (bottom). Symbols represent numerical results averaged over $10^{4}$ realizations of trajectories consisting of $T=2^{23}$ discrete time steps.

with $c_{H}=\Gamma(1+2 H) \sin (\pi H)$. This asymptotic form is depicted by thin solid curves in Fig. 2.

(ii) We see that $\gamma \geq 1$ in the whole range of variation of $\omega$. This signifies that the standard deviation of the single-component single-trajectory PSD always exceeds its mean value. Consequently, the PDF in Eq. (10) is effectively broad, and the analysis of the power spectrum using the standard ensembleaveraged PSD $\mu$ only is rather meaningless.

(iii) A most remarkable feature-rendering $\gamma$ a crucial and highly practical property for FBM-type processes-is that it offers the sought criterion for anomalous diffusion, since the values attained by $\gamma$ in the limit $\omega \rightarrow \infty$ are distinctly different: $\sqrt{2}$, $\sqrt{5} / 2$, and 1 , independent of the exact value of $H$ but solely dependent on whether one has a superdiffusive $(H>1 / 2)$, diffusive $(H=1 / 2)$, or subdiffusive $(H<1 / 2)$ behavior, respectively. These analytical predictions are fully confirmed by numerical simulations for a number of $H$ values.

Before we proceed, it may be expedient to dwell some more on the last point. When dealing with particle-tracking experiments, one often observes values of $\alpha$ that are only slightly different from 1 . Consequently, in these cases, it is not obvious whether one is dealing with anomalous diffusion, or simply if the fitting of the curves started too early and includes transient behavior. On the other hand, the asymptotic value of $\gamma$ at large frequencies provides, in principle, an immediate answer to this question and reveals whether the underlying diffusion process is normal or anomalous. Such an unequivocal confirmation of anomalous diffusion can provide extremely valuable evidence to drive efforts into searching for microscopic 
mechanisms underlying the dynamics and lead eventually to a deeper comprehension of the processes in the system under study.

Note that here, however, we resort to proof-of-concept numerical simulations, because the confirmation of this prediction requires a rather big statistical sample, which we are unable to create in the current experimental analysis. We nonetheless perform such an analysis below in Sec. V (see Fig. 4): It appears to be instructive and shows that even a small sample containing only a few tens of trajectories can provide a meaningful representation of the overall trend. Given the current rapid progress in single-particle tracking techniques, sufficiently large experimental samples are certainly within reach.

Even though $\gamma$ allows us to determine whether the process is subdiffusive or superdiffusive, it does not permit one to deduce the value of the anomalous-diffusion exponent $\alpha$. Below, we discuss how one can find this further piece of the puzzle by analyzing the asymptotic behavior of the ensemble-averaged PSD $\mu$ and the corresponding limiting behavior of the PDF in Eq. (10). Consider first the case of subdiffusion $(H<1 / 2)$. We suppose that $\omega$ is sufficiently large, such that $\gamma-1 \leq \varepsilon$, where $\varepsilon$ is a small parameter. In virtue of relation (11), the above inequality holds for $\omega$ within the interval $\omega \in\left(\omega_{l}^{\text {(sub) }}, \infty\right)$ where $\omega_{l}^{\text {(sub) }}=1 /\left(2 c_{H} \varepsilon\right)^{1 /(1-2 H)}$ (e.g., for $H=1 / 4$ and $\varepsilon=0.01$ one gets $\left.\omega_{l} \approx 6.4 \times 10^{3}\right)$. In this limit, the denominator in Eq. (9) becomes a full square, i.e., $\Phi_{\lambda} \simeq[1+\mu \lambda]^{-k}$, with accuracy set by $\varepsilon$. This means, in turn, that the PDF of $S_{k}(f, T)$ becomes, up to terms of order of $\varepsilon$, the gamma distribution with shape parameter $k$ and scale parameter $\mu$. Consequently, in this case, the $k$-component single-trajectory PSD obeys the equality in distribution

$$
\frac{S_{k}(f, T)}{\mu^{(\mathrm{sub})}} \stackrel{d}{=} A+O(\varepsilon),
$$

where the omitted terms are small in $\varepsilon$, and $A$ is a random numerical factor with distribution

$$
P(A)=A^{k-1} \exp (-A) / \Gamma(k) .
$$

In the superdiffusive case $(H>1 / 2)$, we again assume that $\omega$ is sufficiently large such that the inequality $\sqrt{2}-\gamma \leq$ $\varepsilon$ holds. By virtue of relation (11), this is true when $\omega \in$ $\left(\omega_{l}^{\text {(sup) }}, \infty\right)$ with $\omega_{l}^{\text {(sup) }}=\left(2 \sqrt{2} c_{H} / \varepsilon\right)^{1 /(2 H-1)}$ (e.g., for $H=$ $3 / 4$ and $\varepsilon=0.01$, we have that $\omega_{l}^{\text {(sup) }} \approx 7.1 \times 10^{4}$, i.e., a somewhat bigger value than the one in the subdiffusive case). In this limit, the coefficient in front of the term quadratic in $\lambda$ in the denominator in Eq. (9) [i.e., $\left(2-\gamma^{2}\right)$ ], is less than $\varepsilon$ such that $\Phi_{\lambda} \simeq[1+2 \mu \lambda]^{-k / 2}$ and, in turn, the PDF of $S_{k}(f, T)$ becomes the gamma distribution with scale $2 \mu$ and shape parameter $k / 2$. Consequently, the $k$-component single-trajectory PSD follows the equality in distribution

$$
\frac{S_{k}(f, T)}{\mu^{(\sup )}} \stackrel{d}{=} 2 B+O(\varepsilon)
$$

where $B$ is a random numerical factor with distribution

$$
P(B)=B^{k / 2-1} \exp (-B) / \Gamma(k / 2) .
$$

Therefore, the equalities in Eqs. (12) and (14) suggest that for both the subdiffusive and superdiffusive cases the single-trajectory PSD should always be linearly proportional to its ensemble-average value $\mu$ (which incorporates the dependence on frequency) at large values of $\omega$. The proportionality factor is merely a random number with distribution given by Eqs. (13) or (15), which does not entail any additional dependence on $f$ or $T$.

Below, we specify the spectral content of $\mu$. In the Supplemental Material [54], we show that for subdiffusive FBM at sufficiently large values of $T$ and $f, \mu$ has the scaling form

$$
\mu^{(\mathrm{sub})}=\frac{2 c_{H} D}{f^{2 H+1}} .
$$

In the superdiffusive case $H>1 / 2$, at large $T$ and $f$,

$$
\mu^{(\text {sup })}=\frac{2 D}{f^{2}} T^{2 H-1}+\frac{2 c_{H} D}{f^{2 H+1}}+o(1),
$$

where the Landau symbol $o(1)$ states that the omitted terms vanish as $T \rightarrow \infty$. Result (17) unveils two remarkable features of the ensemble-averaged PSD in the superdiffusive case:

(i) First, regardless of the value of $H$, for large $T$, the frequency dependence has the universal $1 / f^{2}$ form, precisely that of the PSD for standard Brownian motion. Therefore, experimental analyses of the frequency dependence of the PSD in the superdiffusive case may lead to the false conclusion that one deals with Brownian motion $(H=1 / 2)$. Consequently, one should exercise care in interpreting the data in this case: While Brownian motion has a PSD that scales as $1 / f^{2}$, the observation of exclusively such a dependence does not guarantee that one indeed deals with Brownian motion.

(ii) Second, a crucial difference from Brownian motion is the dependence of the amplitude on the observation time $T$. This ageing behavior can be used to distinguish the $T$-independent PSD for Brownian motion from the superdiffusive case: $H$ can be deduced by analyzing the spectrum at some fixed frequency given that one expects $S_{k}(f, T) \sim T^{2 H-1}$.

Lastly, we show that the value of $H$ can be deduced from the spectrum evaluated at zero frequency, 


$$
S_{k}(f=0, T)=\frac{1}{T} \sum_{j=1}^{k}\left(\int_{0}^{T} X_{t}^{(j)} d t\right)^{2}
$$

which represents the sum (divided by $T$ ) of squared areas under the projections of the random curve $X_{t}$ on different axes. In the Supplemental Material [54], we show that the ensemble-averaged PSD at zero frequency is universally (for both subdiffusive and superdiffusive $H$ ) described by

$$
\mu(f=0, T)=\frac{D T^{2 H+1}}{(H+1)}
$$

(see also the result in Ref. [10] with the reset rate set equal to zero). On the other hand, the variance of the singletrajectory PSD obeys (see the Supplemental Material [54]), again for any $H$,

$$
\sigma^{2}(f=0, T)=\frac{2 D^{2} T^{4 H+2}}{(H+1)^{2}} .
$$

This result signifies that the coefficient of variation $\gamma=\sqrt{2}$, regardless of the value of $H$, such that the PDF of $S_{k}(f=0, T)$ is the gamma distribution with scale $2 \mu(f=$ $0, T)$ and shape parameter $k / 2$ for any $T$. As a consequence, $S_{k}(f=0, T)$ obeys exactly the single-trajectory relation in Eq. (14) with the correction term $O(\varepsilon)$ identically equal to zero implying that the Hurst index can be deduced directly from the PSD at zero frequency.
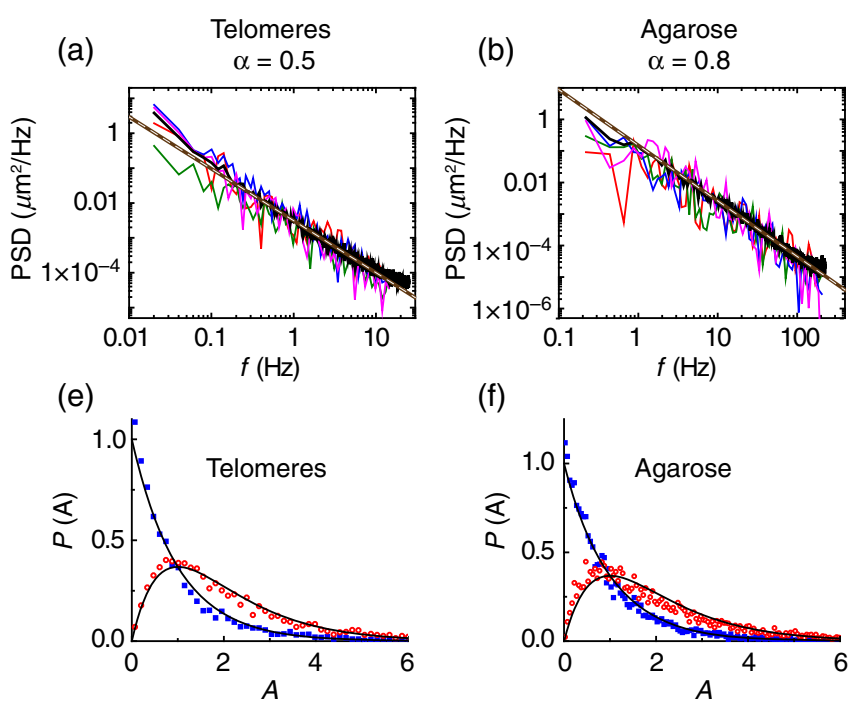

Below, we explore both possibilities to deduce $H$ from a single-trajectory data, taking advantage of the ageing behavior of $S_{k}(f, T)$ and of the dependence of the PSD at zero frequency on the observation time $T$.

\section{COMPARISON WITH EXPERIMENTAL AND NUMERICAL DATA}

We test our predictions for the PSD of single trajectories in four different experimental datasets and multiple numerical simulations. The experimental data consist of two systems exhibiting subdiffusive behavior and two systems exhibiting superdiffusion. For subdiffusive dynamics, we analyze the motion of 50-nm microspheres in agarose gels and telomeres in the nucleus of mammalian cells [56]. For superdiffusive behavior, we study the motion of live amoeba and their intracellular vacuoles. Representative MSDs of individual trajectories in all these systems are presented in Fig. 1 along with their respective averages of the time-averaged MSDs. Examples of PSDs of the single trajectories are shown in Figs. 3(a)-3(d).

The time-averaged MSDs of telomeres scale with an exponent $\alpha=0.5$ (i.e., $H=0.25$ ) for short and intermediate times [56], predicting a PSD $S(f, T) \sim A / f^{1.5}$. As shown in Fig. 3(a), the individual trajectories agree with this prediction, and the ensemble-averaged PSD from 19 trajectories yields $\mu^{\text {(sub) }} \sim 1 / f^{1.49}$. We also show that the experimentally observed fluctuations in the PSDs remarkably confirm the predicted universal distribution Eq. (13) for both one- and two-component PSDs, i.e., for $k=1$ and
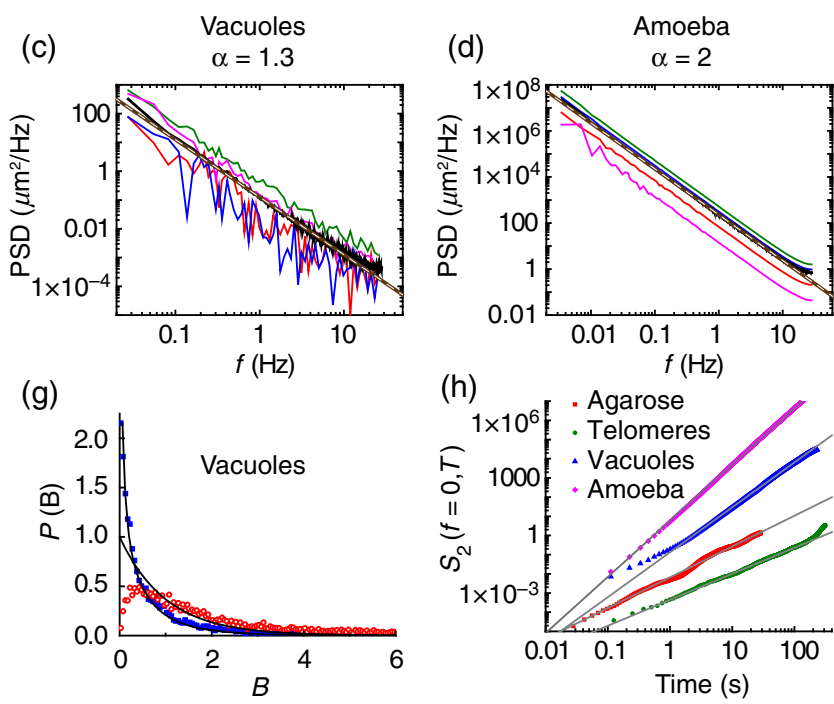

FIG. 3. Power spectrum analysis of experimental datasets. (a)-(d) PSD of representative trajectories along with the ensemble-averaged PSD for telomeres in the nucleus of HeLa cells, 50-nm microspheres in 1.5\% agarose gel, intracellular vacuoles within amoeba, and the motion of amoeba. The dashed thick lines show the $1 / f^{1.49}$ trend for panel (a), $1 / f^{1.76}$ for panel (b), and $1 / f^{2}$ for panels (c) and (d). In each case, the PSDs of four trajectories are presented (log sampled with a factor 1.1 for clarity) together with the ensemble-averaged PSD (thicker black lines, $n=19,20,50$, and 4 trajectories for telomeres, microspheres, vacuoles, and amoeba, respectively). (e)-(g) Distribution of amplitudes of the PSD for one and two components [see Eqs. (13) and (15)]. (h) PSD evaluated at zero frequency. The zero-frequency spectra are shifted for clarity and displayed together with the fitted power-law functions (gray solid lines). The experimental results throughout agree excellently with the theoretical predictions. 
$k=2$, respectively [Fig. 3(e)]. Similar agreements are found for the motion of $50-\mathrm{nm}$ microspheres in $1.5 \%$ agarose gel. As shown in Fig. 1(a), the MSD of these particles scales with an exponent $\alpha=0.87(H=0.43)$ for short times and $\alpha=0.61(H=0.30)$ for long times. The PSD yields $\mu^{\text {(sub) }} \sim 1 / f^{1.76}$ [Fig. 3(b)], and the PSD fluctuations also follow closely a gamma distribution [Fig. 3(f)] as predicted by Eq. (13).

The motion of amoebae and their intracellular vacuoles are good examples of superdiffusive dynamics. Intracellular vacuoles are subdiffusive at short lag times and superdiffusive with $\alpha=1.33$ at long lag times [Fig. 1(b)]. This behavior is typical of active motion in the cytoplasm [62]. Interestingly, the MSDs of the center of mass of the investigated amoebae show almost ballistic motion with $\alpha=1.97$ [Fig. 1(c)]. The PSDs of the motion of both the amoebae and the vacuoles therein clearly show the predicted deceptive $1 / f^{2}$ behavior [Figs. 3(c) and 3(d)]. The distribution of the PSD amplitudes is also shown for the vacuoles in Fig. 3(g) together with the predicted gamma distributions Eq. (15), revealing an excellent agreement for $k=1$. The discrepancy with our two-component analytical prediction for small $B$ values is likely associated with small amplitude antipersistent motion of the vacuoles, as is evident from the trajectories.

In Fig. 3(h), we present the averaged spectra at zero frequency for both subdiffusive and superdiffusive cases [see Eqs. (19) and (20) in Sec. IV]. We use 19, 20, 50 and 4 trajectories for the telomeres, microspheres, vacuoles, and amoeba, respectively, to get directly the Hurst exponents $H=0.30,0.18,0.67$, and 1.92. Despite the small sizes of our statistical samples, the obtained values of $H$ agree well with the values deduced from the corresponding MSDs.

We revisit next the behavior of the coefficient of variation $\gamma$ of the single-trajectory PDF (see Fig. 2) and address the question whether meaningful information can already be drawn from small statistical samples of experimental data. In Fig. 4, we plot the value of $\gamma$ as a function of $\omega=f T$ obtained from only 19 experimentally recorded trajectories of telomeres, 20 trajectories of microspheres in agarose hydrogels, and 50 intracellular vacuole trajectories, as well as from a larger number of trajectories (150) of micrometer-sized beads in an aqueous solution [23]. The microspheres in aqueous solution provide an excellent example of standard Brownian motion, i.e., $H=0.5$. One observes that, indeed, in the large- $\omega$ limit, $\gamma$ converges to distinctly different values for the superdiffusion, normal diffusion, and subdiffusion cases. For vacuoles, at large $\omega$, the coefficient of variation $\gamma$ is observed to converge to $1.55 \pm 0.01$; for Brownian motion (beads in aqueous solution), it is observed to converge to $1.21 \pm 0.01$, for telomeres to $1.05 \pm 0.01$, and for the microspheres in agarose gels to $1.07 \pm 0.01$. In line with our analytical prediction of a universal value of $\gamma$ for subdiffusive FBM, the obtained values for telomeres and microspheres are very

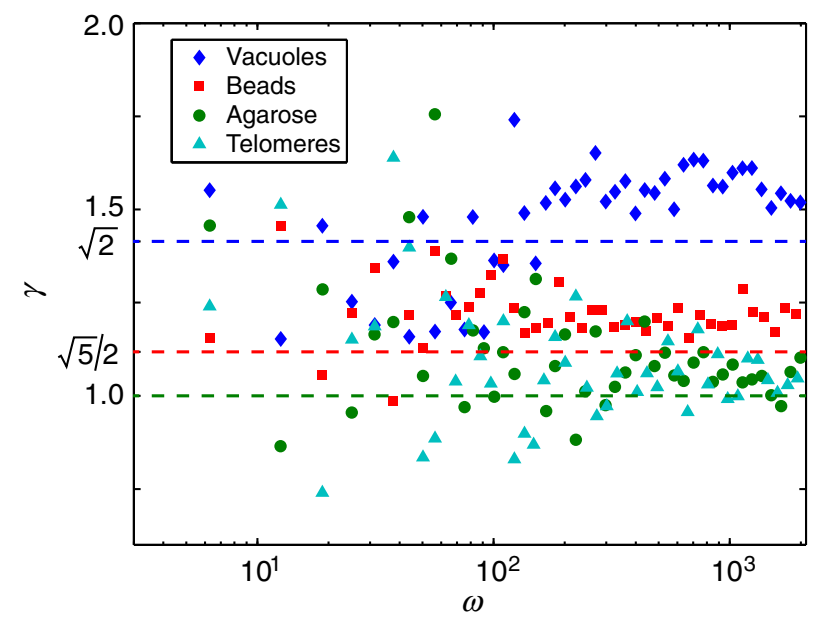

FIG. 4. Coefficient of variation $\gamma$ as a function of $\omega=f T$ in small statistical samples of experimental data. Dashed horizontal lines highlight the analytical predictions made in Sec. IV with blue, red, and green indicating the values $\gamma=\sqrt{2}$ (superdiffusive FBM), $\gamma=\sqrt{5} / 2$ (standard Brownian motion [23]), and $\gamma=1$ (subdiffusive FBM), respectively. Symbols represent the values of $\gamma$ drawn from experiments. Blue diamonds, vacuoles (50 trajectories); red squares, $1.2-\mu \mathrm{m}$ beads in aqueous solution (150 trajectories) [23]; green circles, 50-nm microspheres in agarose hydrogel (20 trajectories); cyan triangles, telomeres in the nucleus of mammalian cells (19 trajectories).

close to each other. Overall, the experimentally determined values for $\gamma$ are only about $10 \%$ larger than our analytical predictions $(\sqrt{2} \approx 1.41$ for vacuoles, $\sqrt{5} / 2 \approx 1.12$ for beads in aqueous solution [23], and 1 for telomeres and microspheres). Given the small size of the statistical sample, we consider such a favorable agreement quite remarkable. In comparison, the perfect agreement of our predictions with $\gamma$ values from FBM simulations (cf. Fig. 2) rather represents an exceptional situation due to the big statistical sample ( $10^{4}$ trajectories). Moreover, in experiments, many different, sometimes uncontrollable factors, e.g., detector noise, may come into play which leads to an increasing variance of the single-trajectory PSD and hence to elevated values of $\gamma$. We plan to examine this important aspect in more detail in our future work.

We further perform extensive analyses of singletrajectory PSDs for different values of $H$ using numerical simulations. Figures 5(a) and 5(b) show the results for single-trajectory PSDs as a function of the frequency (for sufficiently large $T$ ) for different values of $H$ between 1/5 and 4/5. Namely, the subdiffusive cases $(H<1 / 2)$ are shown in Fig. 5(a), and other cases $(H>1 / 2)$ are shown in Fig. 5(b). One observes excellent agreement between the predicted behavior Eqs. (12) and (16), and the numerics even for a small statistical sample consisting of ten realizations. In Fig. 5(c), we also demonstrate that the single-trajectory PSD for a specific subdiffusive case $(H=1 / 3)$ is not ageing. On the other hand, Figs. 5(e) 
(a)

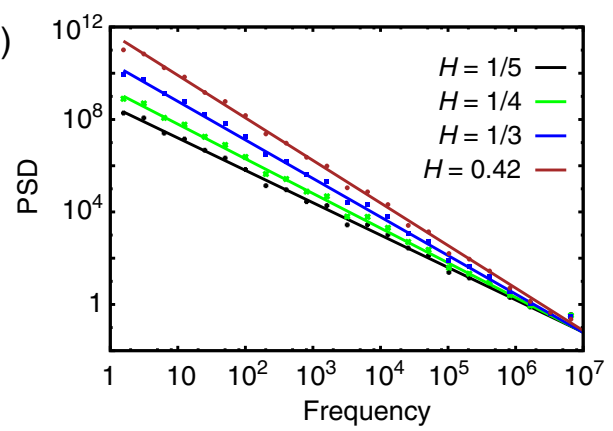

(c)

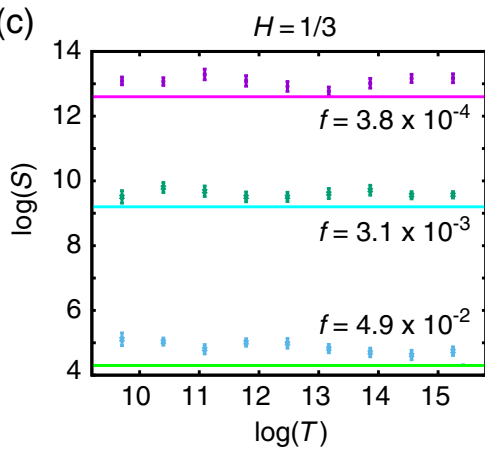

(d)

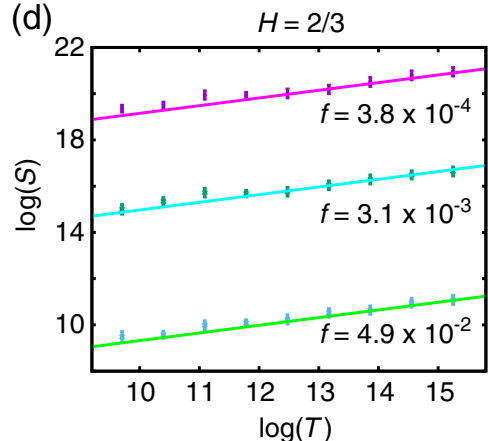

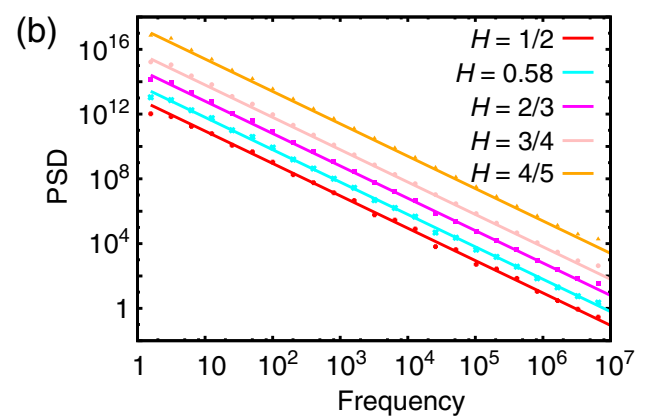

(e)

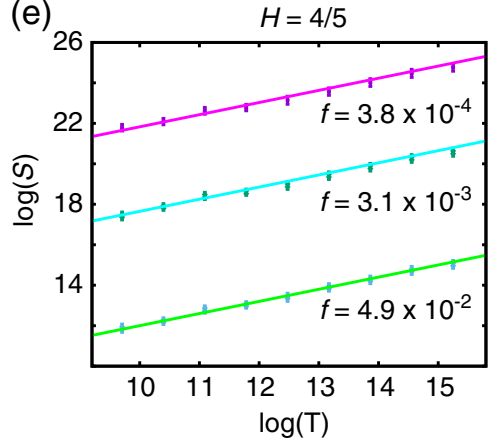

FIG. 5. Numerical confirmation of our theoretical predictions. (a) Frequency dependence of a single-trajectory PSD in the subdiffusive case [Eqs. (12) and (16)] for $H=0.42,1 / 3,1 / 4$, and $H=1 / 5$, averaged over ten realizations. Straight lines have slope $-(2 H+1)$, as shown in Eq. (16). The symbols represent the results of numerical simulations. (b) Deceptive $1 / f^{2}$ dependence [Eq. (17)] in the superdiffusive case. Symbols represent results of numerical simulations for individual trajectories averaged over ten realizations. (c) Apparent independence of a single-trajectory PSD in the subdiffusive case on the observation time for sufficiently large $T$. (d), (e) Ageing behavior of a single-trajectory PSD in the superdiffusive case for three different values of the frequency. Straight lines have slopes $2 H-1$ as shown in Eq. (17). Symbols represent numerical results averaged over 50 realizations.

and 5(f) illustrate the ageing behavior of a single-trajectory PSD for $H=2 / 3$ and $4 / 5$, at three fixed frequencies. Here, the straight lines indicate the predicted ageing dependence $T^{2 H-1}$, Eq. (17), while the symbols represent the results of numerical simulations averaged over 50 realizations. We again observe a perfect agreement with our theoretical predictions.

\section{DISCUSSION}

In summary, here we combine theoretical, numerical, and experimental analyses to provide a comprehensive answer to the conceptually and practically important question: Which information can be reliably obtained from the spectral content of a single realization of naturally occurring anomalous-diffusion processes? Given the widespread occurrence of $1 / f$-type spectra in the analysis of experimental systems and signals across almost every field of physics, such an analysis is very pertinent. Focusing on a wide class of such processes - the so-called fractional Brownian motion-we derive exactly the distribution of a single-trajectory PSD and analyze its asymptotic forms for both subdiffusive and superdiffusive dynamics. On this basis, we unveil several striking features:

(i) At a fixed observation time and in the limit of high frequencies, this distribution reduces to simple forms with a unique scaling given by the ensembleaveraged PSD, which incorporates the full dependence on $f$ and $T$. As a consequence, one expects that for an arbitrary realization of the process, a single-trajectory PSD should exhibit the same large- $f$ dependence as a traditional ensembleaveraged PSD.

(ii) Our experiments and numerical simulations impressively evidence that this $1 / f^{\beta}$ dependence is indeed observed for both super- and subdiffusive FBM-type processes. For subdiffusive processes, the exponent characterizing the spectrum is equal to $2 H+1$, and hence, the anomalous-diffusion exponent can be obtained by evaluating the slope of the PSD. For superdiffusive processes, in contrast, the exponent is deceptively universal and equal to 2 , which can lead to the false conclusion that one deals with ordinary Brownian motion, while in reality the process is superdiffusive. We find this prediction particularly important since it will permit us to avoid a misinterpretation of the experimental results.

(iii) For superdiffusive processes, the amplitude of the PSD is ageing, i.e., dependent on the observation time. However, it is difficult to observe this dependence on a single trajectory since the $T$ dependence is weaker than the large fluctuations between nearby 
frequencies. Here, a statistical sample (comprising, however, only 50 trajectories) is used in order to observe the ageing trend and to extract the value of the anomalous-diffusion exponent from the ageing behavior.

(iv) We show that the coefficient of variation $\gamma$ of a single-trajectory PSD provides a novel criterion for anomalous diffusion. For FBM, its large- $f$ form assumes only three different values depending on whether we observe subdiffusion, normal diffusion, or superdiffusion. Our analytical predictions are in a perfect agreement with the results of numerical simulations for a representative statistical sample ( $10^{4}$ trajectories), but they are also in line with the experimental results obtained from a fairly small statistical sample (19 to 50 trajectories).

(v) Lastly, our theoretical, numerical, and experimental analysis shows unequivocally that the coefficient of variation always exceeds the value 1 , meaning that the standard deviation of a single-trajectory PSD is generically bigger than its mean value. In standard nomenclature of the statistical analysis, the distributions which possess such a property are considered to be effectively broad. This implies that the analysis of the spectral content of individual trajectories in terms of only the ensemble-averaged PSD has limited meaning, which justifies completely our quest for the full PDF of this important characteristic property.

To conclude, from an experimental perspective, our results serve as a reliable framework in the interpretation of noisy data obtained from a single trajectory-it has become routine to garner few individual particle trajectories of impressive length in the wake of superresolution microscopy and supercomputing. In perspective, our results will thus play an important role in extracting more physical information from them.

Finally, we remark that especially in the complex environment of biological cells, where a vast array of specific and nonspecific interactions transpire, FBM does not account, of course, for all possible types of observed anomalous diffusions. Therefore, additional stochastic mechanisms may be superimposed, such as short-time or even simultaneous scale-free trapping time dynamics $[27,28,63,64]$. In other instances, FBM may be tempered or there may occur dynamical transitions between different types of FBM [51,52]. Extensions of our analysis over other possible kinds of anomalous diffusion, such as the FBM models with dynamical transitions [51], "diffusing diffusivity" models [65], scaled Brownian motion [66], or continuous-time random walks with a broad distribution of waiting times [67] are necessary in order to get a full understanding of the behavior of the PSD in experimentally relevant systems. We believe that our work presents an important first step towards such an understanding and will prompt a systematic case-by-case analysis.

\section{ACKNOWLEDGMENTS}

We thank Eli Barkai for discussions. D. K. acknowledges the support of the National Science Foundation under Grant No. 1401432. Research of E. M. is supported in part by the European Research Council under the European Unions Horizon 2020 Research and Innovation Program (Grant Agreement No. 694925). R. M. acknowledges the German Research Foundation (DFG) Grant No. ME-1535/7-1 and a Humboldt Polish Honorary Research Fellowship from the Foundation for Polish Science. N. L. and C. S.-U. thank the DFG for funding through the Collaborative Research Centre CRC 1261 Magnetoelectric Sensors: From Composite Materials to Biomagnetic Diagnostics. M. W. acknowledges financial support by the VolkswagenStiftung (Az. 92738).

D. K. and X. X. performed experimental single-trajectory analyses of anomalous diffusion of microspheres in agarose hydrogels, N. L. and C. S.-U. studied the dynamics of amoebae and their intracellular vacuoles, while L. S. and M. W. performed particle-tracking experiments with telomeres in the nucleus of mammalian cells. D. K. and M. W. analyzed the power spectra of individual experimental trajectories. E. M. performed the numerical analysis of power spectral densities of fractional Brownian motion. R. M., G. O., and A. S. performed all analytical calculations.

D. K., R. M., E. M., G. O., C. S.-U., and M.W. have equally contributed to writing the paper.

The authors declare no conflict of interests.

[1] M. P. Norton and D. G. Karczub, Fundamentals of Noise and Vibration Analysis for Engineers (Cambridge University Press, Cambridge, England, 2003).

[2] R. Voss and J. Clarke, 1/f-Noise in Music and Speech, Nature (London) 258, 317 (1975).

[3] H. Hennig, R. Fleischmann, A. Fredebohm, Y. Hagmayer, J. Nagler, A. Witt, F. J. Theis, and T. Geisel, The Nature and Perception of Fluctuations in Human Musical Rhythms, PLoS One 6, e26457 (2011).

[4] R. O. Weber and P. Talkner, Spectra and Correlations of Climate Data from Days to Decades, J. Geophys. Res. 106, 20131 (2001).

[5] A. Sornette and D. Sornette, Self-Organized Criticality and Earthquakes, Europhys. Lett. 9, 197 (1989).

[6] J. W. Kirchner, X. Feng, and C. Neal, Fractal Stream Chemistry and Its Implications for Containment Transport in Catchments, Nature (London) 403, 524 (2000).

[7] A. A. Balandin, Low-Frequency $1 / f$ Noise in Graphene Devices, Nat. Nanotechnol. 8, 549 (2013).

[8] P. A. Frantsuzov, S. Volkán-Kacsó, and B. Jank, Universality of the Fluorescence Intermittency in Nanoscale Systems: Experiment and Theory, Nano Lett. 13, 402 (2013).

[9] D. Krapf, Nonergodicity in Nanoscale Electrodes, Phys. Chem. Chem. Phys. 15, 459 (2013). 
[10] S. N. Majumdar and G. Oshanin, Spectral Content of Fractional Brownian Motion with Stochastic Reset, J. Phys. A 51, 435001 (2018).

[11] O. Bénichou, P. L. Krapivsky, C. Mejía-Monasterio, and G. Oshanin, Temporal Correlations of the Running Maximum of a Brownian Trajectory, Phys. Rev. Lett. 117, 080601 (2016).

[12] E. Marinari, G. Parisi, D. Ruelle, and P. Windey, Random Walk in a Random Environment and $1 / f$ Noise, Phys. Rev. Lett. 50, 1223 (1983).

[13] D. S. Dean, A. Iorio, E. Marinari, and G. Oshanin, Sampleto-Sample Fluctuations of Power Spectrum of a Random Motion in a Periodic Sinai Model, Phys. Rev. E 94, 032131 (2016).

[14] M. Zorkot, R. Golestanian, and D. J. Bonthuis, The Power Spectrum of Ionic Nanopore Currents: The Role of Ion Correlations, Nano Lett. 16, 2205 (2016).

[15] K. Nørregaard, R. Metzler, C. M. Ritter, K. Berg-Sørensen, and L. B. Oddershede, Manipulation and Motion of Organelles and Single Molecules in Living Cells, Chem. Rev. 117, 4342 (2017).

[16] D. B. Kemp, K. Eichenseer, and W. Kiessling, Maximum Rates of Climate Change Are Systematically Underestimated in the Geological Record, Nat. Commun. 6, 8890 (2015).

[17] B. Tóth, Y. Lempérière, C. Deremble, J. de Lataillade, J. Kockelkoren, and J.-P. Bouchaud, Anomalous Price Impact and the Critical Nature of Liquidity in Financial Markets, Phys. Rev. X 1, 021006 (2011).

[18] M. Niemann, H. Kantz, and E. Barkai, Fluctuations of $1 / f$ Noise and the Low-Frequency Cutoff Paradox, Phys. Rev. Lett. 110, 140603 (2013).

[19] S. Sadegh, E. Barkai, and D. Krapf, $1 / f$ Noise for Intermittent Quantum Dots Exhibits Non-Stationarity and Critical Exponents, New J. Phys. 16, 113054 (2014).

[20] M. Weiss, Single-Particle Tracking Data Reveal Anticorrelated Fractional Brownian Motion in Crowded Fluids, Phys. Rev. E 88, 010101 (2013).

[21] L. Li, E. C. Cox, and H. Flyvbjerg, Dicty Dynamics: Dictyostelium Motility as Persistent Random Motion, Phys. Biol. 8, 046006 (2011).

[22] J. N. Pedersen, L. Li, C. Grâdinaru, R. H. Austin, E. C. Cox, and H. Flyvbjerg, How to Connect Time-Lapse Recorded Trajectories of Motile Microorganisms with Dynamical Models in Continuous Time, Phys. Rev. E 94, 062401 (2016).

[23] D. Krapf, E. Marinari, R. Metzler, G. Oshanin, X. Xu, and A. Squarcini, Power Spectral Density of a Single Brownian Trajectory: What One Can and Cannot Learn From It, New J. Phys. 20, 023029 (2018).

[24] N. D. Schnellbächer and U. S. Schwarz, The Power of a Single Trajectory, New J. Phys. 20, 031001 (2018).

[25] E. Frey and K. Kroy, Brownian Motion: A Paradigm of Soft Matter and Biological Physics, Ann. Phys. (Amsterdam) 14, 20 (2005).

[26] C. Di Rienzo, V. Piazza, E. Gratton, F. Beltram, and F. Cardarelli, Probing Short-Range Protein Brownian Motion in the Cytoplasm of Living Cells, Nat. Commun. 5, 5891 (2014).
[27] J.-H. Jeon, V. Tejedor, S. Burov, E. Barkai, C. SelhuberUnkel, K. Berg-Sørensen, L. Oddershede, and R. Metzler, In Vivo Anomalous Diffusion and Weak Ergodicity Breaking of Lipid Granules, Phys. Rev. Lett. 106, 048103 (2011).

[28] S. A. Tabei, S. Burov, H. Y. Kim, A. Kuznetsov, T. Huynh, J. Jureller, L. H. Philipson, A. R. Dinner, and N. F. Scherer, Intracellular Transport of Insulin Granules is a Subordinated Random Walk, Proc. Natl. Acad. Sci. U.S.A. 110, 4911 (2013).

[29] J.-H. Jeon, N. Leijnse, L. Oddershede, and R. Metzler, Anomalous Diffusion and Power-Law Relaxation in Wormlike Micellar Solution, New J. Phys. 15, 045011 (2013).

[30] J. Szymanski and M. Weiss, Elucidating the Origin of Anomalous Diffusion in Crowded Fluids, Phys. Rev. Lett. 103, 038102 (2009).

[31] I. Bronshtein, E. Kepten, I. Kanter, S. Berezin, M. Lindner, A. B. Redwood, S. Mai, S. Gonzalo, R. Foisner, Y. ShavTal, and Y. Garini, Loss of Lamin A Function Increases Chromatin Dynamics in the Nuclear Interior, Nat. Commun. 6, 8044 (2015).

[32] S. Havlin and D. ben-Avraham, Diffusion in Disordered Media, Adv. Phys. 36, 695 (1987).

[33] G. Afek, J. Coslovsky, A. Courvoisier, O. Livneh, and N. Davidson, Observing Power-Law Dynamics of PositionVelocity Correlation in Anomalous Diffusion, Phys. Rev. Lett. 119, 060602 (2017).

[34] D. Krapf, Mechanisms Underlying Anomalous Diffusion in the Plasma Membrane, Curr. Top. Membr. 75, 167 (2015).

[35] J.-H. Jeon, M. Javanainen, H. Martinez-Seara, R. Metzler, and I. Vattulainen, Protein Crowding in Lipid Bilayers Gives Rise to Non-Gaussian Anomalous Lateral Diffusion of Phospholipids and Proteins, Phys. Rev. X 6, 021006 (2016).

[36] R. Metzler, J.-H. Jeon, and A. G. Cherstvy, Non-Brownian Diffusion in Lipid Membranes: Experiments and Simulations, Biochim. Biophys. Acta 1858, 2451 (2016).

[37] S. Sadegh, J. L. Higgins, P. C. Mannion, M. M. Tamkun, and D. Krapf, Plasma Membrane is Compartmentalized by a Self-Similar Cortical Actin Meshwork, Phys. Rev. X 7, 011031 (2017).

[38] C. K. Peng, J. Mietus, J. M. Hausdorff, S. Havlin, H. E. Stanley, and A. L. Goldberger, Long-Range Anticorrelations and Non-Gaussian Behavior of the Heartbeat, Phys. Rev. Lett. 70, 1343 (1993).

[39] C.-K. Peng, S. V. Buldyrev, A. L. Goldberger, S. Havlin, F. Sciortino, M. Simons, and H. E. Stanley, Long-Range Correlations in Nucleotide Sequences, Nature (London) 356, 168 (1992).

[40] A. W. Rempel, E. D. Waddington, J. S. Wettlaufer, and M. G. Worster, Possible Displacement of the Climate Signal in Ancient Ice by Premelting and Anomalous Diffusion, Nature (London) 411, 568 (2001).

[41] B. B. Mandelbrot and J. W. van Ness, Fractional Brownian Motions, Fractional Noises and Applications, SIAM Rev. 10, 422 (1968).

[42] R. Metzler, J.-H. Jeon, A. G. Cherstvy, and E. Barkai, Anomalous Diffusion Models and Their Properties: NonStationarity, Non-Ergodicity, and Ageing at the Centenary of Single Particle Tracking, Phys. Chem. Chem. Phys. 16, 24128 (2014). 
[43] I. Goychuk, Viscoelastic Subdiffusion: Generalized Langevin Equation Approach, Adv. Chem. Phys. 150, 187 (2012).

[44] J. F. Reverey, J.-H. Jeon, H. Bao, M. Leippe, R. Metzler, and C. Selhuber-Unkel, Superdiffusion Dominates Intracellular Particle Motion in the Supercrowded Space of Pathogenic Acanthamoeba Castellanii, Sci. Rep. 5, 11690 (2015).

[45] S. C. Weber, A. J. Spakowitz, and J. Theriot, Bacterial Chromosomal Loci Move Subdiffusively through a Viscoelastic Cytoplasm, Phys. Rev. Lett. 104, 238102 (2010).

[46] G. R. Kneller, K. Baczynski, and M. Pasenkiewicz-Gierula, Consistent Picture of Lateral Subdiffusion in Lipid Bilayers: Molecular Dynamics Simulation and Exact Results, J. Chem. Phys. 135, 141105 (2011).

[47] J.-H. Jeon, H. M.-S. Monne, M. Javanainen, and R. Metzler, Lateral Motion of Phospholipids and Cholesterols in a Lipid Bilayer: Anomalous Diffusion and Its Origins, Phys. Rev. Lett. 109, 188103 (2012).

[48] M. Di Pierro, D. A. Potoyan, P. G. Wolynes, and J. N. Onuchic, Anomalous Diffusion, Spatial Coherence, and Viscoelasticity from the Energy Landscape of Human Chromosomes, Proc. Natl. Acad. Sci. U.S.A. 115, 7753 (2018).

[49] K. Burnecki, E. Kepten, J. Janczura, I. Bronshtein, Y. Garini, and A. Weron, Universal Algorithm for Identification of Fractional Brownian Motion. A Case of Telomere Subdiffusion, Biophys. J. 103, 1839 (2012).

[50] D. Ernst, M. Hellmann, J. Köhler, and M. Weiss, Fractional Brownian Motion in Crowded Fluids, Soft Matter 8, 4886 (2012).

[51] M. R. Shaebani, Z. Sadjadi, I. M. Sokolov, H. Rieger, and L. Santen, Anomalous Diffusion of Self-Propelled Particles in Directed Random Environments, Phys. Rev. E 90, 030701(R) (2014).

[52] D. Molina-Garcia, T. Sandev, H. Safdari, G. Pagnini, A. Chechkin, and R. Metzler, Crossover from Anomalous to Normal Diffusion: Truncated Power-Law Noise Correlations and Applications to Dynamics in Lipid Bilayers, New J. Phys. 20, 103027 (2018).

[53] J.-H. Jeon, A. V. Chechkin, and R. Metzler, First Passage Behaviour of Fractional Brownian Motion in TwoDimensional Wedge Domains, Europhys. Lett. 94, 20008 (2011).

[54] See Supplemental Material at http://link.aps.org/ supplemental/10.1103/PhysRevX.9.011019 for details of the derivations.
[55] C. Gosse and V. Croquette, Magnetic Tweezers: Micromanipulation and Force Measurement at the Molecular Level, Biophys. J. 82, 3314 (2002).

[56] L. Stadler and M. Weiss, Non-Equilibrium Forces Drive the Anomalous Diffusion of Telomeres in the Nucleus of Mammalian Cells, New J. Phys. 19, 113048 (2017).

[57] R. B. Davies and D.S. Harte, Tests for Hurst Effect, Biometrika 74, 95 (1987).

[58] A. Wood and G. Chan, Simulation of Stationary Gaussian Processes in $[0,1]^{d}$, J. Comput. Graph. Stat. 3, 409 (1994).

[59] C. R. Dietrich and G. N. Newsam, Fast and Exact Simulation of Stationary Gaussian Processes through Circulant Embedding of the Covariance Matrix, SIAM J. Sci. Comput. 18, 1088 (1997).

[60] T. Dieker, Simulation of Fractional Brownian Motion, $\mathrm{Ph} . \mathrm{D}$. thesis, Centrum Wiskunde \& Informatica and University of Twente, 2002, and revised version from 2004.

[61] M. Mandjes and T. Dieker, On Spectral Simulation of Fractional Brownian Motion, Probab. Eng. Inf. Sci. 17, 417 (2003).

[62] P. Bursac, G. Lenormand, B. Fabry, M. Oliver, D. A. Weitz, V. Viasnoff, J. P. Butler, and J. J. Fredberg, Cytoskeletal Remodelling and Slow Dynamics in the Living Cell, Nat. Mater. 4, 557 (2005).

[63] A. V. Weigel, B. Simon, M. M. Tamkun, and D. Krapf, Ergodic and Nonergodic Processes Coexist in the Plasma Membrane as Observed by Single-Molecule Tracking, Proc. Natl. Acad. Sci. U.S.A. 108, 6438 (2011).

[64] A. Weron, K. Burnecki, E. J. Akin, L. Solé, M. Balcerek, M. M. Tamkun, and D. Krapf, Ergodicity Breaking on the Neuronal Surface Emerges from Random Switching between Diffusive States, Sci. Rep. 7, 5404 (2017).

[65] A. V. Chechkin, F. Seno, R. Metzler, and I. M. Sokolov, Brownian Yet Non-Gaussian Diffusion: From Superstatistics to Subordination of Diffusing Diffusivities, Phys. Rev. X 7, 021002 (2017).

[66] J.-H. Jeon, A. V. Chechkin, and R. Metzler, Scaled Brownian Motion: A Paradoxical Process with a Time Dependent Diffusivity for the Description of Anomalous Diffusion, Phys. Chem. Chem. Phys. 16, 15811 (2014).

[67] H. Scher and E. W. Montroll, Anomalous Transit-Time Dispersion in Amorphous Solids, Phys. Rev. B 12, 2455 (1975). 